\title{
Mesurer l'épaisseur des résidus à la surface d'un sol pour estimer leur biomasse
}

\author{
Pascal Thiébeau* \\ INRA, UMR 614 Fractionnement des Agro-Ressources et Environnement (FARE), 2, Esplanade R. Garros, 51100 Reims, France
}

\begin{abstract}
Résumé - Aujourd'hui, les résidus de culture ne sont plus considérés comme des déchets: leur contribution aux équilibres agronomiques et environnementaux des systèmes de culture est reconnue. Dans ce contexte, connaître leur quantité à la surface d'un sol, après une récolte, devient un enjeu important de la gestion des matières organiques dans la rotation des cultures. Généralement, l'estimation quantitative se réalise par prélèvement d'échantillons, afin d'en déterminer la masse sèche (MS) au laboratoire. L'objectif de ce travail est de proposer une méthode rapide de quantification des résidus au sol, dont l'hypothèse est basée sur une méthode d'estimation de biomasse prairiale sur pied. Ce travail a été réalisé sur des exploitations pratiquant l'agriculture de conservation en région de grandes cultures. Les résidus ont été broyés lors de la récolte ou lors d'une opération spécifique suivante. Les données de biomasse ont été collectées à l'aide de cadres de $0,5 \times 0,5 \mathrm{~m}$, tandis que les mesures d'épaisseur ont été effectuées à l'aide d'un aspérimètre. Ce travail compare deux méthodes de mesure d'épaisseur: soit par répétitions aléatoires de positionnement de l'aspérimètre le long d'un transect perpendiculaire au sens de progression de la moissonneuse, soit par répétitions côte à côte. Un test du nombre de mesures d'épaisseur a été réalisé pour connaître l'espacement maximal à conserver pour réaliser une estimation précise. Les résultats montrent qu'il est préférable de réaliser les mesures d'épaisseur des résidus sur l'intégralité d'un transect de moissonneuse pour estimer avec précision cette information, et qu'il faut maintenir 100 mesures d'épaisseur pour conserver une estimation proche de la mesure d'épaisseur de référence, l'espacement entre deux mesures ne devant pas excéder $6 \mathrm{~cm}$. Par ailleurs, ce travail montre qu'il est possible d'obtenir une approximation de la biomasse présente au champ avec une bonne qualité d'estimation, en retenant qu'un millimètre d'épaisseur de résidu représente $50 \mathrm{~g}$ MS.m $\mathrm{m}^{-2}$.
\end{abstract}

Mots clés : résidu de culture / surface du sol / épaisseur / biomasse / estimation

\begin{abstract}
Measuring the thickness of crop residues on the soil surface to estimate their biomass. Today, crop residues are no longer considered as waste: their contributions to the agronomic and environmental balance of cropping systems are recognized. In this context, knowing their quantity on the soil surface after harvest, becomes an important stake of the management of organic matter in the crop rotation. Generally, the quantitative estimation is carried out by taking samples, in order to determine the dry mass (DM) in the laboratory. The aim of this work is to propose a rapid method of quantification of soil residues, the assumption of which is based on a method for estimating standing grass biomass. This work has been carried out on farms practising conservation agriculture in a crop farming region. The residues were ground during the harvest or a subsequent specific operation. Biomass data were collected using $0.5 \times 0.5 \mathrm{~m}$ frames, while thickness measurements were collected using an asperimeter. This work compares two methods of thickness measurement: either by random repetitions of positioning of the asperimeter along a transect perpendicular to the direction of progression of the harvester, or by side-by-side repetitions. A test of the number of thickness measurements was carried out to know the maximum spacing to keep making an accurate estimate. The results show that it is preferable to perform residue thickness measurements over the entire harvester transect to accurately estimate this information, and that 100 thickness measurements must
\end{abstract}

\footnotetext{
*Auteur de correspondance : pascal.thiebeau@inra.fr
} 
be maintained to keep an estimate close to the reference thickness measurement; the spacing between two measurements must not exceed $6 \mathrm{~cm}$. Moreover, this work shows that it possible to approximate the biomass left on the soil with a good quality of estimation by retaining that one millimeter of residue thickness represents $50 \mathrm{~g}$ DM.m ${ }^{-2}$.

Keywords: crop residue / soil surface / thickness / biomass / estimate

\section{Introduction}

Les résidus de culture ont longtemps été considérés comme des déchets dont il fallait se débarrasser pour pouvoir implanter la culture suivante. Or, avec les considérations écologiques liées au réchauffement climatique, ces résidus retrouvent leur intérêt dans les assolements pour leurs actions positives sur le maintien du stock de matières organiques des sols, leur rôle sur la stabilité des sols face aux agressions anthropiques et climatiques, mais également pour le stockage de carbone qu'ils constituent et leur contribution à fournir des nutriments aux cultures suivantes au cours de leur dégradation (Thiébeau et Recous, 2016, 2017). Ainsi, la détermination de la biomasse au sol après une récolte permet de quantifier les masses de carbone présentes, et d'estimer les retours de nutriments que l'on peut en attendre au cours du temps. Cette quantification est souvent appréhendée par une ou plusieurs mesures physiques de la masse des résidus (Oorst et al., 2007 ; Pascault et al., 2010; Ringselle et al., 2014). Cette donnée varie selon l'espèce cultivée et son niveau de production, et fluctue selon les conditions climatiques auxquelles les plantes ont été exposées au cours de leur croissance. La répartition des résidus au sol est rarement homogène: elle dépend des largeurs de coupe des machines, de la qualité de répartition des broyeurs, mais également des conditions climatiques plus ou moins humides ou venteuses au moment de la récolte. La méthode d'estimation de cette masse, mise au point par Thiébeau et Recous (2016), est basée sur la récolte de plusieurs placettes de résidus. Néanmoins, comme d'autres mesures de paramètres biologiques au champ, elle est laborieuse et nécessite le recours à un laboratoire. Cette difficulté de mise en œuvre a été également rencontrée dans l'évaluation de la production sur pied des prairies et a été surmontée par des mesures de hauteur d'herbe (Duru et Bossuet, 1992; Mathieu et Fiorelli, 1985). C'est pourquoi l'hypothèse de construire une relation entre l'épaisseur de résidus et la quantité de biomasse a été testée. En effet, en parvenant à l'établir, il deviendrait possible d'estimer la quantité de biomasse dès le terme de la mesure d'épaisseur au champ.

L'objectif du travail présenté est de mettre au point cette relation (mesure de l'épaisseur des résidus au champ-quantité de biomasse présente).

\section{Matériels et méthode}

La mise au point de la méthode d'estimation de l'épaisseur des résidus a été réalisée au cours des étés et automnes 2009, 2010 et 2011. Les exploitations et les cultures support de cette étude sont décrites dans l'article de Thiébeau et Recous (2016): il s'agit d'exploitations pratiquant les techniques culturales sans labour (TCSL) depuis plusieurs années. Les cultures support de cette étude sont le blé (Triticum aestivum L.), le colza (Brassica napus subsp. napus forma napus L.), l'escourgeon (Hordeum vulgare subsp. hexastichum L.), le maïs grain (Zea mays L.), l'orge de printemps (Hordeum vulgare L.), le pois protéagineux (Pisum sativum L.) et le tournesol (Helianthus annuus L.). Les résidus, après récolte, correspondent aux restes aériens des plantes, composés des tiges, feuilles, restes d'épis, capitules, rafles, menue paille, etc. Ils sont à l'état broyé, soit par le broyeur incorporé aux machines de récolte, soit à l'aide d'un broyeur de paille à axe horizontal pour les résidus de colza, maïs et tournesol. Dans ce cas, l'agriculteur intervient dans les 24 à $48 \mathrm{~h}$ qui suivent la récolte.

Les mesures de biomasse et d'épaisseur des résidus de culture sont réalisées après ces étapes, c'est-à-dire 24 à 72 h après chaque récolte, sur un transect perpendiculaire au sens de la récolte correspondant à une largeur de récolteuse (Thiébeau et Recous, 2016).

\subsection{Mesure de l'épaisseur des résidus}

La mesure de l'épaisseur des résidus de culture est réalisée à l'aide d'un aspérimètre conçu pour des mesures aux champs (Thiébeau et al., 2011). Il comprend un train de 55 tiges en fibre de verre et polyester, espacées de $2 \mathrm{~cm}$ (Fig. 1A). Chaque tige exerce une pression au sol de $0,125 \mathrm{~kg} . \mathrm{cm}^{-2}$ qui permet de respecter l'architecture du paillis et des résidus les plus fragiles.

L'outil est positionné à l'endroit choisi pour la mesure d'épaisseur des résidus. Le train de tiges qui le compose est maintenu en position «haute » à l'aide d'une règle coulissante munie de targettes à ses extrémités. Le déverrouillage de la règle libère le train de tiges, que l'on descend délicatement, verticalement, jusqu'à l'appliquer à la surface des résidus. La position millimétrique de chaque tige, en épaisseur, est relevée sur une fiche d'enregistrement par lecture des graduations du papier millimétré placé derrière elle.

L'épaisseur des résidus est obtenue en deux lectures : une première lecture consiste, après libération des tiges, à noter les valeurs dites «hautes» des tiges; puis une seconde lecture consiste, après avoir relevé le train de tiges, retiré délicatement les résidus de la surface du sol sans déplacer l'outil, et libéré de nouveau le train de tiges, à en recueillir les valeurs «basses ». L'épaisseur des résidus sous chaque tige est obtenue par différence entre sa valeur «haute» et sa valeur «basse».

Deux méthodes, notées A et B, ont été mises en œuvre, soit de façon indépendante pour optimiser le temps de mesure au champ, soit de façon complémentaire afin de disposer d'un jeu de données suffisant pour comparer les deux méthodes d'estimation sur la valeur d'épaisseur obtenue. Pour la méthode $\mathrm{A}$, la mesure de l'épaisseur des résidus a été réalisée en positionnant l'aspérimètre de façon aléatoire en deux endroits d'une même largeur de coupe; tandis que pour la méthode $\mathrm{B}$, il a été placé dans deux positions côte à côte sur l'intégralité de la largeur de coupe (Fig. 1A). Dans cette configuration, l'outil est positionné à une extrémité de la 


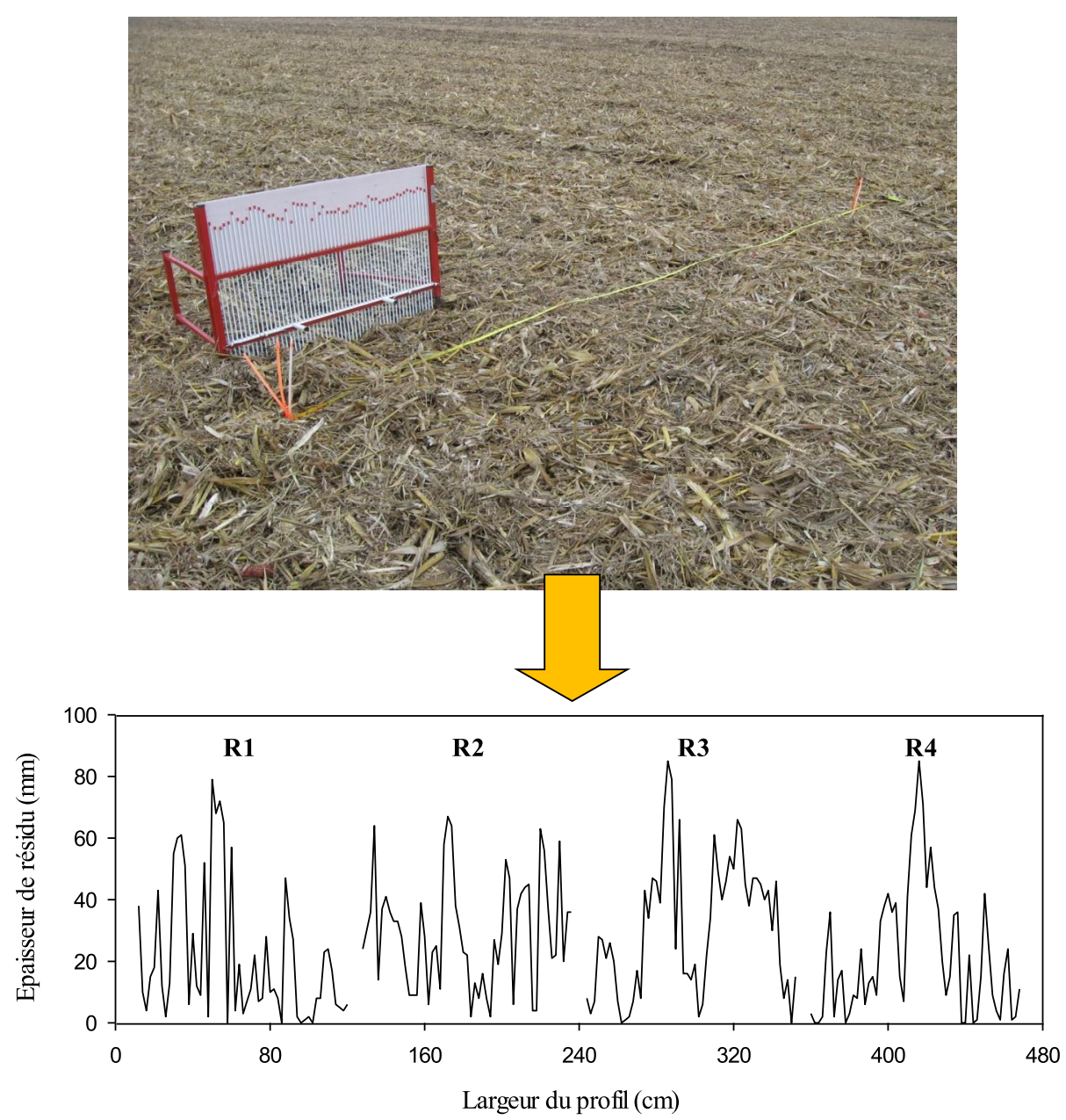

Fig. 1. Méthodologie d'utilisation d'un aspérimètre pour caractériser l'épaisseur des résidus sur une largeur de récolteuse $(4,80 \mathrm{~m})$ par répétitions côte à côte (R1 à R4) (Thiébeau et al., 2011).

Fig. 1. Methodology for using an asperimeter to characterize the thickness of residues over a width of reaper (4.80 m) by side-by-side repetitions (R1 to R4) (Thiébeau et al., 2011).

largeur du transect à caractériser. Après lecture du premier profil de la série, il est déplacé de façon à ce que le pied de l'outil du second profil coïncide précisément avec l'empreinte du pied pour les mesures du premier profil. On calcule ensuite une moyenne de l'ensemble de ces relevés élémentaires (méthode A ou B) pour constituer une seule valeur d'estimation d'épaisseur des résidus du transect.

\subsection{Relation (épaisseur de résidus-quantité de matière sèche totale de résidus)}

La base de données issue du travail de Thiébeau et Recous (2016) a été le support de cette investigation. L'unité de prélèvement pour l'estimation de la matière sèche totale (MST) des résidus est constituée d'un cadre de dimensions intérieures de $0,5 \times 0,5 \mathrm{~m}$. Les prélèvements comprennent plusieurs cadres unitaires, constituant chacun des placettes de prélèvement dont le nombre dépend de la largeur de coupe de la récolteuse. Il en est prélevé par mètre linéaire de largeur de coupe. Dans l'exemple montré à la figure $1 \mathrm{~A}$, cinq placettes de prélèvement ont été réalisées car la largeur de récolte était de $4,80 \mathrm{~m}$.
En pratique, la mesure de l'épaisseur du transect de largeur de récolte complet est réalisée en premier lieu; puis, derrière cette ligne de mesure, la récolte des placettes de résidus. Le décalage opéré est de $0,5 \mathrm{~m}$ au maximum, afin de minimiser la variabilité spatiale des résidus entre l'estimation de la MST et la mesure de son épaisseur.

\subsection{Espacement à conserver entre deux mesures d'épaisseur}

La mesure de l'épaisseur n'étant pas automatisée, la collecte de sa valeur tous les deux centimètres d'un même transect est consommatrice de temps. L'impact de l'espacement de mesure a été étudié afin d'en connaître l'effet sur la qualité des résultats obtenus. Compte tenu du petit nombre de mesures d'épaisseurs réalisées par la méthode A (une centaine), seules les données collectées par la méthode B ont fait l'objet de cette analyse. Pour chaque situation présentée, l'épaisseur de résidus obtenue en faisant la moyenne de toutes les valeurs d'épaisseur d'un transect (épaisseur de référence) a été comparée aux moyennes obtenues en évinçant 
les mesures d'une tige sur $2,3,4,5,10$ et 20 d'un transect complet, correspondant aux espacements de mesure de $4,6,8$, 10,20 et $40 \mathrm{~cm}$.

\subsection{Traitements statistiques des données}

Toutes les moyennes présentées sont accompagnées de leur erreur d'estimation standardisée (SE) afin de pouvoir être comparées entre elles.

Les ajustements linéaires et non linéaires ont été réalisés à l'aide du programme statistique de Sigma-Plot 12 (Systat Software, 2010) après validation de la normalité des variables par le test Shapiro-Wilk. Les critères d'évaluation des ajustements sont l'erreur quadratique au modèle (RMSE) et le coefficient de détermination $\left(R^{2}\right)$. Ils sont définis comme suit:

$$
\mathrm{RMSE}=\sqrt{\frac{1}{n} \cdot \sum_{i=1}^{n}\left(P_{i}-O_{i}\right)^{2}}
$$

$O$ et $P$ sont respectivement les valeurs observées et prédites, $n$ est le nombre de paires observées-simulées. La RMSE s'exprime dans la même unité que la grandeur physique considérée. Elle est souvent exprimée en valeur relative de la valeur moyenne de référence (rRMSE), en pour cent.

$R^{2}$ est le carré de la corrélation du coefficient de Pearson, entre les valeurs observées et les valeurs simulées. Il décrit la proportion de la variance totale des données observées qui peut être expliquée par le modèle. Il est calculé comme suit:

$$
R^{2}=\left[\frac{\sum_{i=1}^{n}\left(P_{i}-\bar{P}\right) \cdot\left(O_{i}-\bar{O}\right)}{\sigma_{P} \cdot \sigma_{O}}\right]^{2},
$$

avec $\bar{P}$ et $\bar{O}$ comme valeurs moyennes respectivement estimées et observées.

Le seuil de signification maximal retenu est $P<0,05$. Pour les intervalles de confiance (IC) et de prédiction (IP) présentés, le seuil de signification retenu est de $95 \%$.

\section{Résultats}

\subsection{Relation (épaisseur de résidus-MST)}

Les données recueillies au cours des trois campagnes de mesures font état de 14 couples (épaisseur de résidus-MST) acquis par la méthode A, 14 couples acquis par la méthode B, et 11 couples en appliquant les deux méthodes sur un même champ, que l'on désignera comme «mixtes", notées Am et Bm. L'ensemble de ces mesures totalise 39 couples (épaisseur de résidus-MST), soit 3 pour l'escourgeon et le tournesol, 4 pour l'orge de printemps, 6 pour le colza et le pois, 8 pour le blé et 9 pour le maïs.

\subsubsection{Comparaison des méthodes $A$ et $B$ de mesure de l'épaisseur des résidus}

Cette comparaison est réalisée à partir des 11 couples Am et $\mathrm{Bm}$ mesurés simultanément sur les mêmes champs. Les cultures concernées, blé, colza, escourgeon, maïs, pois et tournesol correspondent à des épaisseurs de mulch se distribuant de 5 à $26 \mathrm{~mm}$ (Fig. 2A), proches de la première

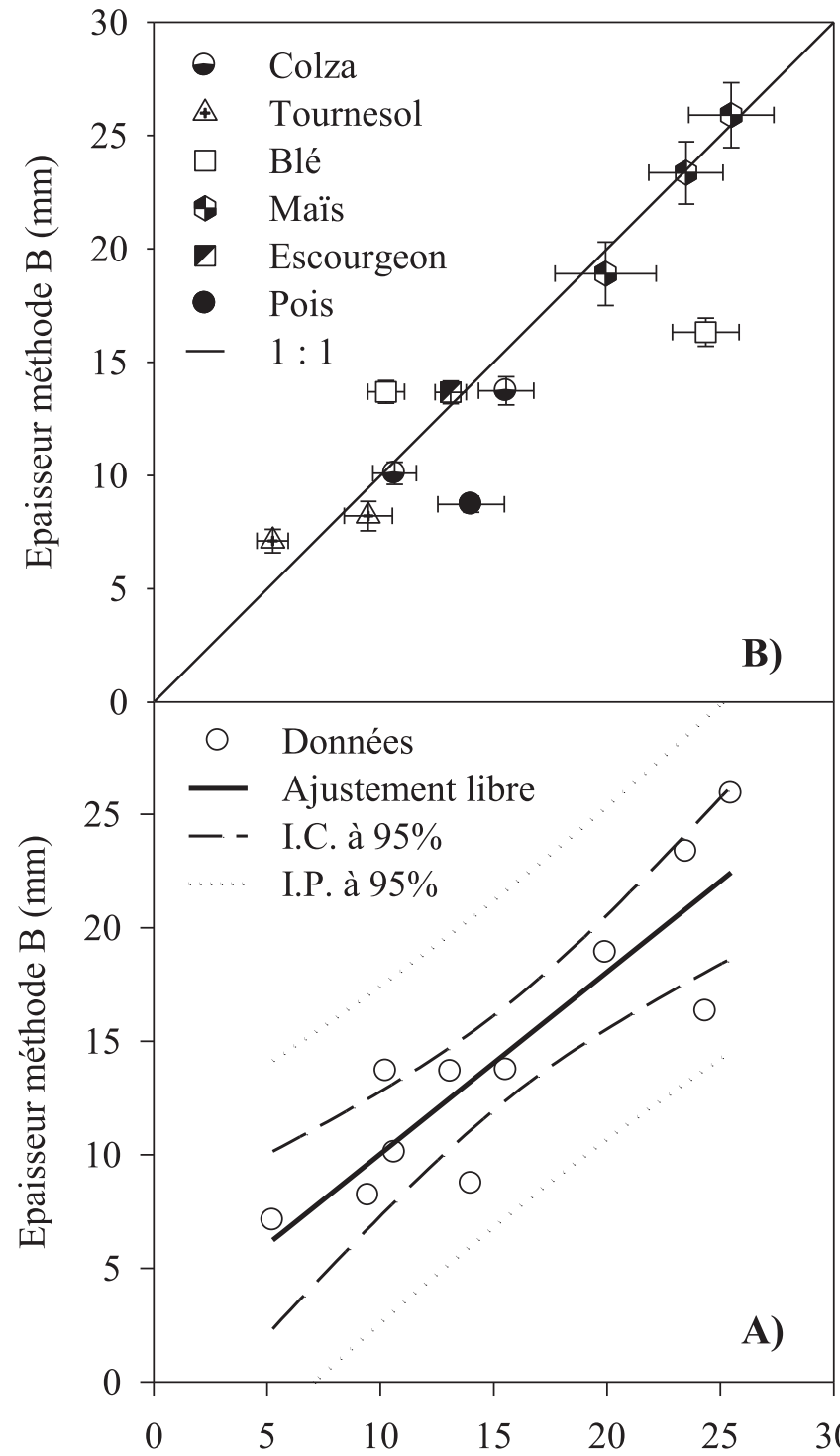

Epaisseur méthode A ( $\mathrm{mm})$

Fig. 2. Comparaison des deux méthodes de mesure d'épaisseur des résidus: par positionnements aléatoires en deux emplacements d'une même largeur de récolteuse (méthode A) ou par positionnements côte à côte sur l'intégralité d'une largeur de récolteuse (méthode B). A) Données accompagnées des tracés de l'ajustement libre, des intervalles de confiance et de prédiction; B) Distribution et identification des cultures accompagnées de leur erreur standard autour de la bissectrice.

Fig. 2. Comparison of two methods for measuring the thickness of residues: by random positioning at two locations on the same harvester width (method A) or by positioning side-by-side on the whole harvester width (method B). A) Data accompanied by the plots of free fit, confidence intervals and prediction; B) Distribution and identification of crops with their standard error around the bisector.

bissectrice (Fig. 2B). L'analyse de variance ne montre pas de différence entre les deux méthodes de mesures $(P=0,56)$, l'année de mesure $(P=0,51)$ ou d'interaction entre méthode et année de mesure $(P=0,43)$. Néanmoins, l'épaisseur estimée 
par la méthode $\mathrm{A}(15,64 \mathrm{~mm})$ est supérieure à celle de la méthode $\mathrm{B}(13,94 \mathrm{~mm})$, présente des coefficients de variation d'estimation d'erreur $(8,8 \%)$ supérieurs à la méthode $\mathrm{B}$ $(5,3 \%)$, avec des médianes qui sont respectivement de 7,9 et $4,9 \%$. La méthode B paraît donc plus précise pour estimer l'épaisseur des résidus, même si aucune différence significative n'apparaît. Une correction d'estimation de la mesure d'épaisseur par la méthode A peut être proposée, pour se rapprocher des mesures de la méthode $\mathrm{B}$, à l'aide d'un modèle de type linéaire de la forme:

$$
Y=a \cdot X+b .
$$

Les valeurs de sa pente $a=0,801$ et de sa constante $b=2,036$ $\left(R^{2}=0,7800 ; \quad \mathrm{RMSE}=2,8 \mathrm{~mm} ; \quad P<0,001\right)$ permettent de corriger les données de façon à ce que les points de mesure se distribuent de part et d'autres de l'ajustement (Fig. 2A). Néanmoins, compte tenu du faible nombre de mesures et de l'absence de différence statistique entre les deux méthodes, cette correction ne sera pas appliquée pour la suite du travail présenté.

\subsubsection{Impact de la composition des mulchs et des espèces sur la qualité des données collectées}

Les quantités de résidus, ainsi que leurs compositions assez différentes, pouvant avoir un impact sur la relation épaisseurMST, il a été procédé à des analyses de variances pour les deux variables étudiées, MST et épaisseur de résidus, selon les deux méthodes (A et B) de mesure d'épaisseur utilisées.

Il existe un effet «espèce» sur la MST mesurée $(P<0,001)$, sans effet de la méthode de mesure de l'épaisseur $(P>0,05)$, avec maïs $\geq$ blé $\geq$ (colza, escourgeon $) \geq$ (tournesol, orge de printemps, pois).

Par rapport aux mesures d'épaisseur, il existe un effet «méthode de mesure» $(P=0,03)$ et «espèce» $(P<0,001)$, avec maïs $\geq$ escourgeon $\geq$ (blé, colza, pois) $\geq$ (orge de printemps, tournesol). En revanche, il n'y a pas d'interaction entre «méthode de mesure de l'épaisseur» et «espèce» $(P=0,95)$. Quelle que soit la culture, l'estimation de l'épaisseur des résidus par la méthode A place les points de mesure sous la première bissectrice au regard d'une estimation réalisée par la méthode B (Fig. 3).

\subsubsection{Relation (épaisseur de résidus-MST)}

La relation (épaisseur de résidus-MST) est établie en conservant les 25 couples de données où la mesure de l'épaisseur est réalisée sur l'intégralité d'une largeur de récolteuse (méthode $\mathrm{B}$ et $\mathrm{Bm}$ ), auxquels les 14 couples de la méthode A sont ajoutés sans correction de leur valeur. En ajustant librement ces 39 données, la valeur de la pente $a$ est de 43,3 $\mathrm{g}$ de MST et celle de la constante $b$ de $111,0 \mathrm{~g}$ avec $R^{2}=0,6885^{* * *}, \mathrm{RMSE}=152,1 \mathrm{~g}, \mathrm{rRMSE}=20,2 \%$. Ce résultat est «très hautement significatif » $(P<0,001)$. La distribution des données, accompagnée du tracé de l'ajustement, des intervalles de confiance (traits d'union) et de prédiction (pointillés) est présentée par la figure 4A.

En forçant l'ajustement par l'origine (Fig. 4B), la valeur de la pente $a$ est de $49,9 \mathrm{~g} \mathrm{MST}$, avec $R^{2}=0,6703 * * *, \mathrm{RMSE}=$ $158,6 \mathrm{~g}, \mathrm{rRMSE}=20,8 \%$; ce qui ne dégrade pas la qualité statistique précédente $(P<0,001)$. L'erreur associée à la valeur de $a$ de l'ajustement libre présente une variation d'estimation

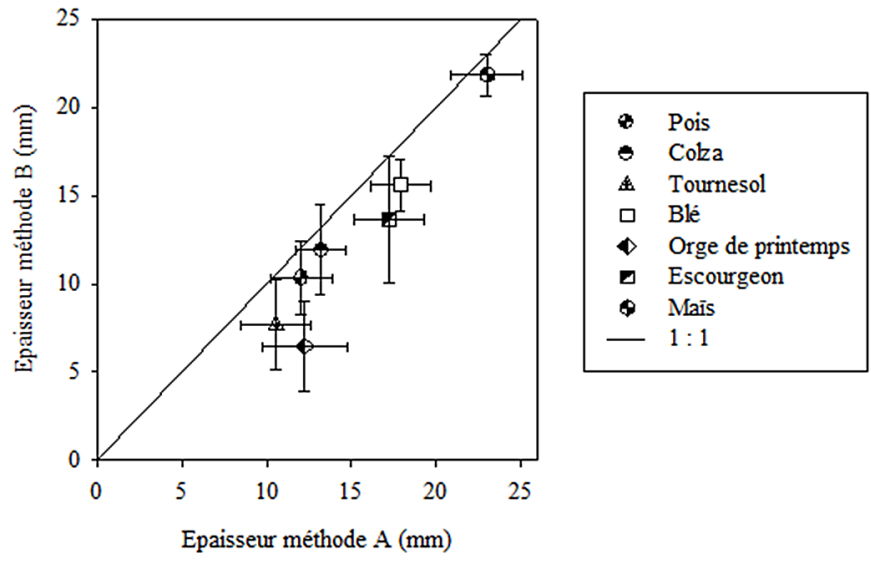

Fig. 3. Comparaison des épaisseurs moyennes de résidues obtenues pour chaque culture selon la méthode de mesure retenue, avec leur erreur standard.

Fig. 3. Comparison of average thicknesses of the residues obtained for each crop according to the chosen measurement method, with their standard error.

de $11,1 \%$, tandis qu'elle est de $3,2 \%$ dans le cas de l'ajustement forcé par l'origine.

Les analyses de variance sur les facteurs MST et épaisseur des résidus (Fig. 4C) montrent un effet « espèce » $(P<0,001)$, avec maïs $\geq$ (blé, colza, escourgeon $) \geq$ (orge de printemps, pois, tournesol). L'ajustement libre présente une valeur de pente $a$ de 47,5 g MST et une constante $b$ de $6,5 \mathrm{~g}(P<0,01)$. La valeur de $a$ est de 47,9 g MST en forçant l'ajustement par l'origine. Celui-ci conserve la même qualité statistique avec $P<0,01$, mais améliore significativement l'erreur associée à la pente dont le coefficient de variation est de 5,4\% au lieu de 20,4\% dans le cas de l'ajustement libre.

\subsection{Espacement de mesure d'épaisseur entre deux tiges d'aspérimètre}

L'analyse se limite aux données acquises par la méthode B et $\mathrm{Bm}$. Les résultats sont présentés par les figures $5 \mathrm{~A}-5 \mathrm{~F}$. Ils montrent que l'augmentation de l'espacement entre deux mesures augmente la dispersion des points de mesures autour de la bissectrice, témoin d'une dégradation de la qualité d'estimation vis-à-vis de l'épaisseur de référence. Néanmoins, pour les deux premières situations, une mesure tous les $4 \mathrm{~cm}$ (Fig. 5A) et tous les $6 \mathrm{~cm}$ (Fig. 5B), les nuages de points restent proches de la bissectrice. Ce constat est confirmé par la qualité des critères statistiques des ajustements linéaires. Le $R^{2}$ se dégrade dès la suppression d'1 mesure sur 2 (espacement de $4 \mathrm{~cm}$ ), et plus nettement à partir de 4 mesures sur 5 (espacement de $10 \mathrm{~cm}$ ). Cette tendance est confirmée par la RMSE. La rRMSE dépasse le seuil de $5 \%$ dès l'espacement de mesure de $8 \mathrm{~cm}$. Cependant, ils restent «très hautement significatifs » $(P<0,001)$.

\section{Discussion}

Le choix de la méthode de mesure de l'épaisseur des résidus se révèle important pour estimer la quantité de résidus 


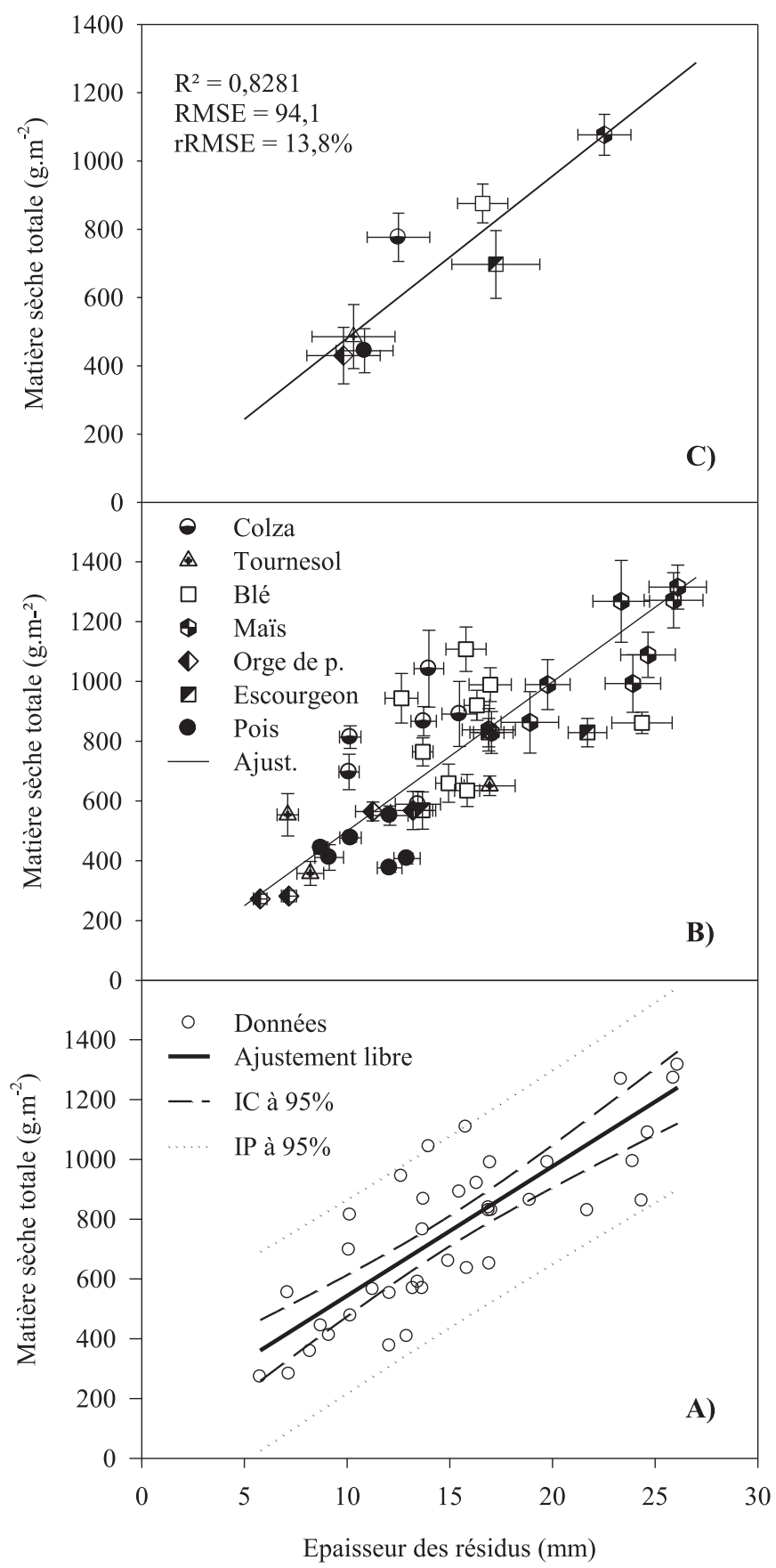

Fig. 4. Relation entre l'épaisseur de résidus $(\mathrm{mm})$ et la quantité de matière sèche totale de résidus présents à la surface du sol $\left(\mathrm{g}\right.$ MST.m $\left.{ }^{-2}\right)$. A) Données accompagnées des tracés de l'ajustement libre, des intervalles de confiance et de prédiction; B) Distribution et identification des cultures accompagnées de leur erreur standard autour de l'ajustement forcé par l'origine; C) Distribution et identification des moyennes par espèce accompagnées de leur erreur standard autour de l'ajustement libre.

Fig. 4. Relation between the measured thickness ( $\mathrm{mm}$ ) and the amount of total dry matter residues on the soil surface $\left(\mathrm{g} T D M . \mathrm{m}^{-2}\right)$. A) Data accompanied by the plots of free fit, confidence intervals and prediction; B) Distribution and identification of crops accompanied by their standard error around forced adjustment by origin; $C$ ) distribution and identification of means by species accompanied by their standard error around free adjustment.

au champ aussi près que possible des mesures physiques. Ainsi, réaliser les mesures sur une largeur de récolteuse complète (méthode B) se révèle plus précis que de procéder par positionnement aléatoire de l'aspérimètre en deux endroits de ce transect (méthode A). La variabilité d'estimation observée reflète la variabilité de répartition spatiale des résidus au sol (Thiébeau et Recous, 2016), davantage que le délai d'intervention pour réaliser les mesures au champ, compris entre 24 et $72 \mathrm{~h}$ après chaque récolte. L'estimation de perte de masse par rapport à la quantité initiale, réalisée à partir des 


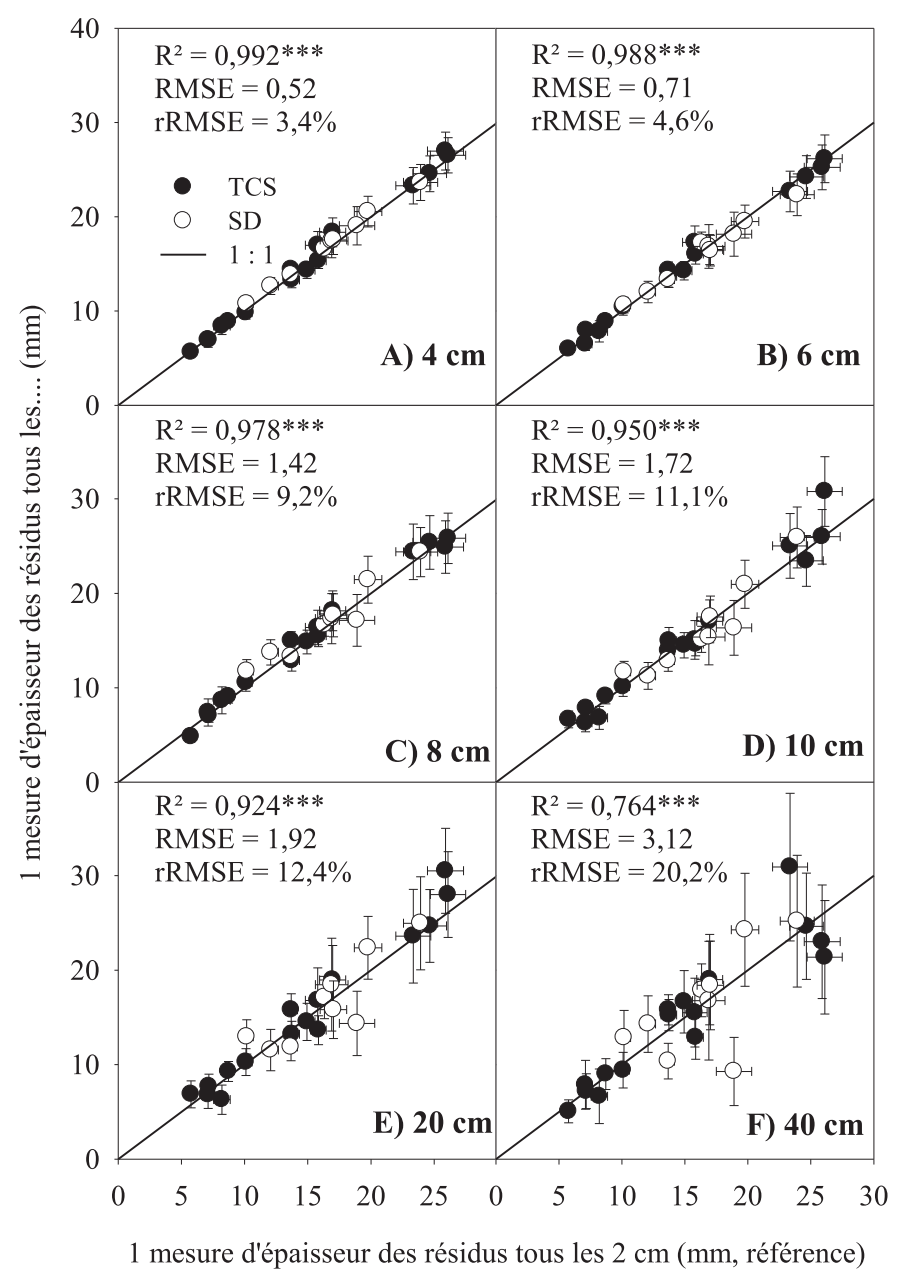

Fig. 5. Incidence de la réduction du nombre de mesures d'épaisseur des résidus par rapport à la mesure de référence. De $\mathrm{A}$ ) à $\mathrm{F}$ ) réalisation d'une mesure tous les $4,6,8,10,20$ et $40 \mathrm{~cm}$.

Fig. 5. Impact of reducing the number of residues thickness measurements from the reference measurement. From A) to F) making a measurement every 4, 6, 8, 10, 20 and $40 \mathrm{~cm}$.

travaux de Thiébeau et Recous (2017), montre qu'elle est de 5 à $7 \%$ au maximum, ce qui interfère peu avec l'ensemble des mesures. En effet, d'une part, l'aspérimètre est utilisé à une précision millimétrique, sans effet de l'opérateur ni de son déplacement (Thiébeau et al., 2011), d'autre part, et de ce fait, si perte de masse il y a, elle est prise en compte lors des mesures d'épaisseur qui sont réalisées le jour même de l'estimation de la MST.

Les résultats montrent qu'il faut conserver un nombre significatif de mesures élémentaires, réalisées sur un transect complet de récolteuse, pour estimer convenablement l'épaisseur des résidus. Si l'on retient un risque d'erreur maximum de $5 \%$, il faut maintenir un intervalle maximum de 4 à $6 \mathrm{~cm}$ entre deux mesures d'épaisseur pour conserver une bonne estimation de sa valeur. Au-delà, les résultats obtenus montrent une nette dégradation par rapport à la mesure de référence réalisée à intervalles de $2 \mathrm{~cm}$. En retenant un intervalle moyen de $5 \mathrm{~cm}$ entre deux mesures, cela signifie qu'il ne faut pas moins de 100 mesures élémentaires pour estimer convenablement l'épaisseur de résidus d'un champ qui aurait été récolté avec une moissonneuse disposant d'une barre de coupe de $5 \mathrm{~m}$ de largeur. Même si cela nécessite de passer un peu plus de temps, le fait de collecter davantage de données au champ, sur un transect complet, permet d'améliorer significativement la précision d'estimation de l'épaisseur de résidus $\left(R^{2}>0,80\right)$.

Pour estimer la biomasse de résidus au champ à partir de la mesure de son épaisseur, il parait préférable de retenir un ajustement forcé par l'origine, puisqu'en l'absence de résidu, la valeur de biomasse au sol est de zéro. L'utilisation de cette relation évite le recours à un laboratoire qu'imposeraient les mesures physiques de résidus pour en estimer les masses. Les ajustements permettent d'obtenir une valeur de $a$ de $49,9 \mathrm{~g}$ MST $\left( \pm 1,6 \mathrm{~g}\right.$ MST.m $\left.^{-2}\right)$ pour l'ajustement global des 39 mesures élémentaires (Fig. 4B), et de 47,9g MST $\left( \pm 2,6 \mathrm{~g} \mathrm{MST} . \mathrm{m}^{-2}\right)$ pour l'ajustement établi à partir des mesures moyennes par espèce (Fig. 4C). En arrondissant cette valeur à 50 , cela signifie alors qu' $1 \mathrm{~mm}$ d'épaisseur de résidu équivaut à $50 \mathrm{~g}$ de MST.m ${ }^{-2}$, soit $0,5 \mathrm{t} \mathrm{MST.ha}{ }^{-1}$. Cette relation présente donc un intérêt pratique et rapide d'estimation au champ de la biomasse présente au sol. Elle peut être utilisée en sens inverse : en ayant mesuré la biomasse, on peut en estimer l'épaisseur rapidement; ce qui peut également représenter un intérêt dans le cadre de modèles de décomposition de résidus de culture où cette donnée est pour 
le moment une variable optimisée (Galdos et al., 2010), ou de modèles qui étudient les flux d'eau et de chaleur avec le sol (Bussière et Cellier, 1994). Compte tenu des travaux publiés par Thiébeau et Recous (2017), cela s'avèrera également utile pour sensibiliser et inciter les agriculteurs à estimer euxmêmes la dégradation de ces résidus, afin de prédire la restitution d'éléments nutritifs, notamment d'azote, pour en tenir compte dans leurs plans de gestion prévisionnelle à la parcelle.

Ce travail a été réalisé pour des situations de résidus récoltés à maturité. Pour réaliser une estimation sur des résidus frais, comme des cultures intermédiaires broyées par exemple, il sera nécessaire de revoir ces valeurs car une cellule végétale turgescente, en croissance, comporte des parois à la fois souples et de volume différent, par rapport à une cellule mature et déshydratée, ce qui impact son volume, et donc son épaisseur.

\section{Conclusion}

Ce travail original permet d'estimer la quantité de résidus présents au sol après une récolte, à partir de mesures d'épaisseur des résidus. Cette estimation est réalisée avec une bonne précision. On peut se contenter de la réaliser à partir d'un seul transect linéaire (perpendiculaire au sens de la récolte) correspondant à une largeur de récolteuse. Il faut cependant veiller à réaliser au moins une mesure tous les $6 \mathrm{~cm}$ au plus pour conserver une précision suffisante de son estimation, et, a fortiori, d'estimation de la MST au sol. Ce travail montre également qu'un millimètre d'épaisseur de résidu peut être rapproché d'une valeur correspondant à $50 \mathrm{~g} \mathrm{MST.m}^{-2}$.

Remerciements. L'auteur remercie Messieurs Acker, Ferté, Gubelin, Lemey et Martens, exploitants agricoles qui ont accepté de mettre leurs champs à sa disposition afin que cette étude puisse être conduite. Il remercie Akhtar Iqbal et Francis Millon pour leur aide technique. L'agence nationale de la recherche (ANR) est remerciée pour le soutien financier apporté de 2009 à 2013 au programme Systerra, projet
PEPITES (Processus écologiques et processus d'innovations techniques et sociales en agriculture de conservation).

\section{Références}

Bussière F, Cellier P. 1994. Modification of the soil temperature and water content regimes by a crop residue mulch: Experiment and modelling. Agricultural and Forest Meteorology 68: 1-28.

Duru M, Bossuet L. 1992. Estimation de la masse d'herbe par le «sward-stick». Premiers résultats. Fourrages 131: 283-300.

Galdos MV, Cerri CC, Cerri CEP, Paustian K, Van Antwerpen R. 2010. Simulation of sugarcane residue decomposition and aboveground growth. Plant \& Soil 326: 243-259.

Mathieu A, Fiorelli JL. 1985. Utilisation d'un herbomètre pour l'interprétation du déroulement d'un pâturage. Fourrages 101: 3-29.

Oorst K, Merckx R, Grehan E, Labreuche J, Nicolardot B. 2007. Determinants of annual fluxes of $\mathrm{CO}_{2}$ and $\mathrm{N}_{2} \mathrm{O}$ in long-term notillage and conventional tillage systems in northern France. Soil \& Tillage Research 95: 133-148.

Pascault N, Nicolardot B, Bastian F, Thiébeau P, Ranjard L, Maron PA. 2010. In situ dynamics and spatial heterogeneity of soil bacterial communities under different crop residue management. Environmental Microbiology 60: 291-303.

Ringselle B, Bergkvist G, Aronsson H, Andersson L. 2014. Undergrown-sown cover and post-harvest mowing as measures to control Elymus repens. European Weed Research Society 55: 309-319.

Systat Software. 2010. SigmaPolt 12. Disponible sur https:// systatsoftware.com/products/sigmaplot/sigmaplot-details.php (dernière consultation le 09/01/2019).

Thiébeau P, Recous S. 2016. Méthode pour quantifier les biomasses de résidus de récolte à la surface des sols après la moisson. Cahiers Agricultures 25: 45001. DOI: 10.1051/cagri/2016027.

Thiébeau P, Recous S. 2017. Dynamiques de décomposition des résidus de cultures sur des exploitations pratiquant l'agriculture de conservation en région Grand-Est, France. Cahiers Agricultures 26: 65001. DOI: 10.1051/cagri/2017050.

Thiébeau P, Millon F, Beaudoin N. 2011. Conception d'un aspérimètre pour mesures aux champs. Les Cahiers des Techniques de l'INRA 72: 37-58.

Citation de l'article : Thiébeau P. 2019. Mesurer l'épaisseur des résidus à la surface d'un sol pour estimer leur biomasse. Cah. Agric. 28 : 11. 